\title{
LAS INTERRUPCIONES DEL EMBARAZO EN LA PRÁCTICA OBSTÉTRICA: RECURSO TERAPÉUTICO VS ABORTO PROVOCADO
}

\begin{abstract}
Mauricio Besio Rollero ${ }^{1}$
Resumen: En el tema del aborto provocado, en general, y del aborto llamado "terapéutico" en particular, se involucran valores importantes para los individuos y para toda la población. Existe mucha confusión respecto de los términos empleados para nominar las distintas acciones que los médicos obstetras deciden realizar en las situaciones clínicas que presentan sus pacientes. Este trabajo pretende precisar cuáles interrupciones de un embarazo corresponden a acciones no solamente lícitas sino obligatorias para un médico, por corresponder a los fines de la medicina, y cuáles son abortos provocados. Para ello se analiza el fin de la profesión médica y a quienes debe el profesional de la salud otorgar siempre sus cuidados. Realiza una reflexión sobre la diferencia entre concepto, criterios y signos para distinguirlos cuando hablamos de viabilidad fetal y de aborto provocado. Desde esa perspectiva, establece el concepto de aborto provocado, buscando sus rasgos necesarios y evitando la confusión con los criterios y signos utilizados para reconocer esa realidad en un caso particular. Finalmente, deduce los criterios y signos de una interrupción de un embarazo en armonía con los valores de la medicina.
\end{abstract}

Palabras clave: ética médica, aborto inducido, interrupción del embarazo, aborto terapéutico

Discontinuation of pregnancy in obstetric practice: therapeutic resource versus provoked abortion

\begin{abstract}
The issue of induced abortion, in general, and the called therapeutic abortion in particular, has generated a lot of concern for both the medical profession, as well as for all society. They involve important values for individuals and for the entire population. There is much confusion about the terms used to nominate the various actions that the obstetricians decide to implement in clinical situations that their patients present. This paper aims to clarify which pregnancy interruptions are not only permissible but obligatory actions to a doctor, and correspond to the goals of medicine, and which of them are really induced abortions. To do so, the purpose of the medical profession is analyzed and to whom the health professional should always provide their care. Performs a reflection on the difference between concept, criteria, and signs to distinguish them when talking about fetal viability and induced abortion. From that perspective, establishes the concept of induced abortion, seeking its necessary features and avoiding confusion with the criteria and signs used to recognize this reality in a particular case. Finally, follows which are the criteria and signs of a termination of pregnancy in agreement with the values of medicine.
\end{abstract}

Key words: medical ethics, induced abortion, pregnancy interruption, therapeutic abortion

\section{As interrupçóes da gravidez na prática obstétrica: recurso terapêutico vs aborto provocado}

Resumo: No tema do aborto provocado, em geral, e do aborto chamado "terapêutico" em particular, se envolvem valores importantes para os individuos e para toda a população. Existe muita confusão a respeito dos termos empregados para nominar as distintas açóes que os médicos obstetras decidem realizar nas situaçóes clínicas que apresentam as suas pacientes. Este trabalho pretende precisar quais interrupçóes de uma gravidez correspondem a ações não somente lícitas senão obrigatórias para um médico, por corresponder aos fins da medicina, e quais são abortos provocados. Para isso se analisa o fim da profissáo médica e a quem deve o profissional da saúde outorgar sempre os seus cuidados. Realiza uma reflexão sobre a diferença entre conceito, critérios e sinais para distingui-los quando falamos de viabilidade fetal e de aborto provocado. A partir dessa perspectiva, estabelece o conceito de aborto provocado, buscando seus traços necessários e evitando a confusão com os critérios e sinais utilizados para reconhecer essa realidade num caso particular. Finalmente, deduz os critérios e sinais de uma interrupção de uma gravidez em harmonia com os valores da medicina.

Palavras-chave: ética médica, aborto induzido, interrupção da gravidez, aborto terapêutico

\footnotetext{
${ }^{1}$ División de Obstetricia y Ginecología, Pontificia Universidad Católica de Chile, Chile Correspondencia: besio@med.puc.cl
} 


\section{Introducción}

El debate sobre el aborto provocado no es indiferente para las personas. Tensiona y compromete a toda nuestra sociedad. No podría ser de otra manera, ya que en él participan valores o principios que no son banales y nos involucran profundamente como individuos y como organización social. En una perspectiva más global, puede interesar como un elemento decidor del tipo de sociedad en la que queremos vivir, o para establecer políticas públicas de asignación de recursos sanitarios o regulación poblacional. En un sentido más particular, involucra directamente a individuos en decisiones que afectan radicalmente a otros, como también a ellos mismos. Ambas esferas son importantes; en la primera las consecuencias de las decisiones comprometen a un gran número de personas y al "carácter" de una sociedad; en el segundo, la afectación es personal.

En todos estos niveles estamos comprometidos los médicos y, de manera más directa, los que nos dedicamos a la atención de la mujer embarazada. Todos podemos actuar en forma organizada y con una perspectiva global al participar como miembros de la sociedad científica que nos agrupa, y actuamos a la vez en forma personal en cada una de nuestras decisiones clínicas, cada vez que interactuamos con nuestros pacientes.

Parece entonces indispensable que, como médicos, participemos activamente en este debate, no solo como interlocutores válidos por la experiencia práctica y el conocimiento científico que poseemos, sino porque somos nosotros los que atendemos a las pacientes que sufren las situaciones críticas que podría abrir la posibilidad de un aborto, y somos nosotros mismos los que tendríamos que efectuar los procedimientos para llevarlo a cabo. Esa decisión y ejecución nos involucra personalmente, ya que el que se decide a ejecutar u omitir una intervención se convierte en un agente con responsabilidad ética, ya que es causa de algún resultado.

El dilema debemos enfrentarlo en forma directa y clara, con un esfuerzo racional y reflexión profunda, para lograr precisar las acciones que nos corresponde realizar imperativamente como médicos obstetras, cuáles no nos corresponde realizar y en cuáles tenemos incertidumbres(1).

\section{Nuestros pacientes}

Lo primero que tenemos que dilucidar es a quién debemos nosotros nuestros cuidados. ¿Cuál es el objetivo, fin o meta que perseguimos como especialidad médica o, dicho de otra forma, qué nos distingue de otros especialistas?

Si somos médicos nos corresponde la salud de nuestros pacientes. La medicina, desde tiempos inmemoriales, se considera como aquella actividad que busca prevenir y curar las enfermedades. Ese es el único objeto, fin o meta de la medicina, y frente a cada intento de otorgarle otros fines u objetivos debemos volver necesariamente a los principios y valores inherentes e inmanentes de la profesión $(2)^{2}$.

Si a la cardiología, como especialidad médica, le corresponden las patologías del sistema cardiovascular, a la obstetricia las del embarazo, parto y puerperio; y a la medicina materno fetal, como subespecialidad de ella, le corresponde el tratamiento del feto in utero y aquellas patologías maternas en cuanto lo afectan. A cada subespecialidad o especialidad le compete un aspecto específico de la medicina, aunque es obvio que todas ellas deben buscar el fin propio de ella, ya que de lo contrario dejarían de ser especialidades médicas. Todas ellas entonces buscan la salud de sus pacientes en sus campos más específicos.

Aceptando el objeto de la medicina, es entonces contrario a la profesión médica atentar contra la vida de un paciente. Cuesta mucho concebir que en una actividad alguien busque un resultado que vaya en contra del objetivo de ella misma. Así como sería incomprensible que un profesor busque que un alumno no aprenda, o que un piloto intente no llegar a su destino, también resulta difícil de comprender que un médico intente afectar la salud o la vida de un paciente a su cargo. Aunque es muy cierto que intervenciones médicas pueden tener como resultado un daño e inclu-

\footnotetext{
2 The Hastings Center, centro importante de bioética, reunió a representantes de trece países en 1996 para volver a discutir los fines de la medicina. Finalmente, el trabajo propuso mantener los fines tradicionales de ella: la prevención de la enfermedad y de las lesiones, junto con la promoción y mantenimiento de la salud; el alivio del dolor y del sufrimiento causado por la enfermedad y las dolencias; la asistencia y curación de los enfermos, además del cuidado de los que no pueden ser curados, y evitar la muerte prematura y velar por una muerte en paz.
} 
so la muerte de un paciente, estos son efectos no deseados, inevitables o por último debidos a un actuar negligente, pero esos efectos nunca están en la intención del médico.

\section{El aún no nacido como paciente}

Se pueden hacer varias objeciones. La primera y más relevante es sobre el estatuto de paciente del embrión o feto. Claro, la conclusión "el médico respeta la vida del feto", derivada de las premisas "el médico respeta la vida de todo paciente" y "el feto es un paciente", se invalida si la segunda premisa del silogismo es falsa. Si no hay paciente, no hay obligación ética de respetar su integridad ni buscar su salud.

Si bien es cierto que es fácil captar en un niño o adulto aquella interioridad que nos orienta para fundamentar el respeto que merece, debemos reconocer que esa captación se dificulta $-\mathrm{y}$ muchas veces se oscurece por completo- en las primeras etapas de la vida humana. El embrión humano permanece alejado del alcance de una adecuada consideración, dificultad que se debe a varios factores:

$\mathrm{Al}$ ser nuestra primera aproximación hacia la realidad una aproximación mediante los órganos de los sentidos, nuestra primera captación de otro ser humano es a través de lo semejante. Captamos como humano algo que se nos parece, y todo ser humano cuya figura humana se desdibuja se nos presenta como algo distinto. Es necesario por lo tanto un esfuerzo de la inteligencia para captar en ese viviente intrauterino a un individuo de nuestra especie.

Es preciso aceptar que esas primeras etapas de la existencia están bastante fuera de nuestro alcance sensitivo. A diferencia del desarrollo humano desde el nacimiento hacia adelante, al cual estamos habituados a observar día a día, lo que ocurre al interior de la madre no se presenta fácilmente a nuestros sentidos, incluso ella misma solo siente a su hijo en etapas bastante avanzadas del embarazo, y solamente en algunas de sus cualidades sensibles, como el crecimiento y movimiento. La ultrasonografía ha posibilitado apreciar al embrión dentro del útero materno, pero la imagen entregada está muy alejada de como captan natu- ralmente nuestros sentidos y es difícil entenderla sin una capacitación previa.

No tenemos experiencia propia de esas primeras etapas vitales, así como tenemos de la niñez o adultez. Solo tenemos referencia de nuestro nacimiento y primeros ańos de vida por el relato de nuestros familiares. Esa falta de vivencias de esos primeros estadios de nuestra existencia probablemente facilite la consideración de los embriones y fetos humanos como seres extraños.

Además de esas apreciaciones propias de la experiencia, la dificultad más radical se debe a que aquella realidad individual, a la cual pertenecen la intimidad, la inteligencia, la libertad y la capacidad de realizar actos propios, y que nos caracteriza como humanos, no existe en el embrión sino como potencialidad, y luego no puede ser reconocida como algo perteneciente en ese momento a él. Debemos por tanto ser capaces de reconocer solo en el orden de un grupo de células o en un organismo a veces desemejante en el aspecto a nosotros, el ser de un sujeto cuya naturaleza se está recién desplegando $(3)^{3}$.

Muchas veces entonces se nos hace difícil reconocer que el embrión es una persona, constituido por una interioridad de naturaleza racional o inteligente, es decir humana. Desde que aparece a la existencia en algún momento después de la unión de ambos gametos, de la madre y del padre, esa naturaleza racional se va desplegando en el tiempo, constituyendo progresivamente los órganos necesarios para ejercer plena y actualmente su inteligencia y libertad, facultades que en un comienzo existen solo en estado de posibilidad. El hecho de no ejercer las operaciones propiamente humanas, por tenerlas en estado potencial, no determina que no exista un sujeto de naturaleza racional. Eso solo indica que las operaciones más propias del hombre y que lo especifican como tal, no se expresan por falta de desarrollo de los órganos corporales necesarios para el ejercicio de aquellas funciones.

Es justamente la naturaleza personal del embrión lo que funda el estatuto de paciente para él. No

\footnotetext{
3 "...la persona es una sustancia individual de naturaleza racional". Boecio. Sobre la persona y las dos naturalezas, cap. 3 .
} 
se necesita una petición de ayuda de su parte para que un médico le otorgue los cuidados que requiere y asegure que su desarrollo se complete adecuadamente, o para que, expuesto a una situación de riesgo o enfermedad, le realice las acciones médicas necesarias para su mejoría. Un médico obstetra que ha logrado aprehender la bondad poseída por el ser humano desde su aparición a la existencia, podrá ser el médico que, enfrentado a una mujer embarazada, considere en ese mismo instante que tiene a dos pacientes: la madre, que habitualmente le solicita sus cuidados para ella y para su hijo, y ese hijo que es su paciente aún sin solicitarlo, ya que no lo puede hacer.

De lo anterior se desprende la incoherencia de toda acción realizada por un médico cuyo fin sea provocar la muerte del embrión, u otra que arriesgue innecesariamente su existencia. La naturaleza personal de este, su condición de vulnerabilidad y su imposibilidad de solicitar ayuda lo convierten en uno de los seres humanos más necesitados de la acción de un médico(4).

\section{Interrupciones del embarazo}

Parece necesario analizar, en el contexto de las acciones terapéuticas, las diversas acciones que un médico puede realizar en una mujer embarazada, intentar determinar en qué consisten y cuáles son las implicancias éticas de cada una.

En primer lugar hay que decir que la interrupción de un embarazo es una acción que busca que el proceso normal de una gestación no alcance su término natural. Entendida así, como concepto general, su calificación ética va a depender de lo que realmente se está haciendo en cada caso.

Tenemos interrupciones del embarazo absolutamente lícitas, realizadas a diario en todas la maternidades del mundo como herramienta terapéuti$\mathrm{ca}$, destinadas a proteger la salud de la madre, del recién nacido o de ambos. Son aquellas realizadas frente a una patología materna o fetal que indica al médico que es mejor adelantar el parto por algún problema de salud ${ }^{4}$. Habitualmente se realizan cuando el feto está suficientemente maduro

\footnotetext{
${ }^{4}$ Ejemplos de esas situaciones pueden ser la presencia de cicatrices en el útero que inclinen al médico a realizar una cesárea antes que se inicien las contracciones de parto o la evidencia de que el feto no se está alimentando apropiadamente por alguna disfunción placentaria.
}

para vivir fuera del útero y no representan ningún problema ético, ya que buscan siempre el bienestar de ambos.

Existen sin embargo otras situaciones en que la posibilidad de interrumpir un embarazo provocaría en el médico tratante, en la madre y en los familiares un dilema, ya no de carácter técnico, sino de un orden totalmente distinto, que los obligaría a una reflexión sobre los principios y fines que guían su actuar. Actualmente se nombran como posibles razones los problemas psicológicos transitorios de la mujer embarazada, las angustias y trastornos graves por gestaciones producto de violación, los embarazos con fetos con patologías de diversa gravedad, o las patologías maternas que van desde leves, moderadas, hasta las con riesgo vital.

Frente a tan variadas causales invocadas, debemos distinguir cuáles serían las que se adecuan a los fines de la medicina, siendo por tanto obligatorias para un médico realizar — pues constituyen parte de las acciones propias de la especialidad-y cuáles serían aquellas que un médico debiera realizar nunca, puesto que constituyen verdaderamente lo que debemos entender por "aborto provocado".

\section{Aborto: concepto, criterios y signos}

Para la discusión actual intentaremos precisar cuáles de las interrupciones de un embarazo, derivadas de las motivaciones descritas, constituyen lo que habitualmente se entiende y entenderemos como "aborto provocado o inducido", y cuáles son las interrupciones de un embarazo que un médico debiera siempre realizar, ya que están destinadas a cumplir los objetivos de la profesión médica, es decir, buscar la salud de sus pacientes. Para ello será necesario describir y fundamentar tanto lo que el médico realmente hace en cada una de esas situaciones como el sentido de los términos que empleamos, y si ellos se correlacionan adecuadamente con lo que el médico realmente está haciendo.

Es importante señalar lo que se entiende por "aborto", palabra que expresa significados diversos, de connotación ética indiferente o negativa. Hablamos en el lenguaje médico de "aborto" cuando se pierde una gestación "naturalmente" y 
también cuando se lo provoca voluntariamentes. En segundo lugar, cuando se define "aborto provocado" a menudo se confunde el concepto o la idea que se quiere denotar con esa expresión con los criterios y signos que hemos establecido para reconocerlo como tal en una situación particular.

La idea, concepto o definición es algo abstracto, y su elucidación es una tarea estrictamente filosófica. Un criterio concretiza la idea en un mundo físico y su determinación correspondería a la filosofía y a la ciencia, y un signo o test verifica la realización concreta del criterio, y en su determinación y realización concreta participarían la ciencia empírica y la práctica(5). Tanto los signos como los criterios pueden tener distintos grados de sensibilidad y/o especificidad para demostrar su validez en detectar la presencia de la idea en una realidad concreta. Por ejemplo, no toda alza de transaminasas en la sangre (signo) nos indica una infiltración de polimorfonucleares en el intersticio hepático (criterio) provocado por una inflamación hepática o hepatitis (idea). Tampoco toda sustracción de algo ajeno (criterio) es un hurto (idea).

Entendemos como "aborto": "la interrupción de un embarazo antes de la viabilidad fetal". La idea, concepto o definición de viabilidad fetal es "la capacidad de un recién nacido de sobrevivir fuera del útero materno". Los criterios que ocupamos actualmente para determinar si un feto o recién nacido es viable son: una edad gestacional igual o mayor a 23 semanas o un peso igual o mayor a 500 gr. Los signos son varios, entre ellos unas ciertas medidas realizadas mediante ultrasonografía o una medición del tiempo transcurrido desde la última menstruación de la mujer.

La definición, concepto o idea no ha cambiado en el tiempo y difícilmente lo hará. Siempre hemos entendido y siempre entenderemos lo mismo por viabilidad. Sin embargo, los criterios sí cambian, en este caso han cambiado y probablemente cambiarán aún más a medida que el avance del conocimiento médico y las aplicaciones tecnológicas lo permitan. No hace mucho el criterio de viabilidad

\footnotetext{
${ }^{5}$ En el idioma inglés, se habla de abortion, para referirse al aborto provocado y de miscarriage, (pérdida) para referirse a lo que en espańol denominamos aborto espontáneo. Es notable que las pacientes generalmente denominan pérdida a los abortos espontáneos.
}

eran 28 semanas o 1.000 gr. de peso; y ya en algunos lugares se habla de 20 semanas de gestación. Por otro lado, también los signos han cambiado; hace 40 ańos los únicos signos que teníamos eran el tiempo de amenorrea y la medición de la altura uterina, con sensibilidades y especificidades muy diferentes al test ecográfico actual.

Lo anterior tiene una relevancia no menor para el análisis ético de las acciones. El lenguaje debe reflejar adecuadamente la realidad de esas situaciones y las definiciones deben apuntar lo más rigurosamente posible a lo que se entiende por lo que se intenta definir. Si definimos por ejemplo -con los criterios actuales y como algunos lo hacen- el aborto provocado como "la interrupción de un embarazo antes de las 23 semanas o antes de que el feto pese 500 gr" y la viabilidad fetal también en esos rangos de tiempo gestacional y peso, toda interrupción antes de ese tiempo de gestación o ese peso sería un aborto provocado. Sería por tanto ilegal en nuestro país y éticamente ilícito, ya que se estaría intentando la muerte de ese feto. La realidad es distinta y, desde la perspectiva del actuar médico, en muchas situaciones clínicas nosotros realizamos interrupciones del embarazo comprendidas en lo expresado en esas definiciones, que no son ilegales en nuestro país y tampoco vulneran nuestras obligaciones hacia nuestros pacientes.

Los criterios y signos tienen una utilidad operacional, nos sirven para tomar decisiones. No podemos decidir con los conceptos, ya que estos tienen existencia solo en nuestra mente. No existe la viabilidad fetal, no existe la capacidad de sobrevivir fuera del útero. Lo que existen son fetos que al nacer sobreviven y otros que no lo hacen. Nosotros observamos todos esos casos individuales y, en una inicial abstracción de todos ellos, establecemos que existe una relación entre su edad gestacional y su peso con la sobrevida fuera del útero; a mayor edad gestacional y a mayor peso existe mejor posibilidad de sobrevivir. En una abstracción más profunda, concebimos algo original, la idea de viabilidad, que nos permite entender de lo que hablamos cuando nos referimos a esa capacidad que tienen algunos fetos de sobrevivir.

Es evidente para nuestras observaciones que esa capacidad es progresiva y gradual, y que, de- 
pendiendo de los cuidados que otorguemos a los recién nacidos, habrá posibilidad de potenciarla. Sin embargo, la consideración de los costos de sufrimiento, emocionales y pecuniarios, ponderados con las posibilidades de éxito, nos hace razonable intentar esfuerzos terapéuticos de distinta intensidad en cada caso particular, juicio conocido en ética médica como juicio de proporcionalidad(6): a algunos con mayor posibilidad de éxito, les ofreceremos toda la capacidad tecnológica disponible, a otros parte de esa capacidad y a otros solo los cuidados dignos mínimos.

Para ello nos sirven los criterios y los signos: para reconocer la presencia de aquellos en cada caso particular. Es teóricamente posible que un recién nacido de 22 semanas o quizá de menos edad sobreviva si le aplicamos todos los recursos tecnológicos disponibles, y es perfectamente imaginable que eso será cada vez más probable en algunos decenios más. También sabemos que algunos sobre esa edad gestacional no lo harán, pero en un aquí y ahora tenemos que hacer un corte, y el que tenemos son 23 semanas y 500 gr. Para reconocer que un feto dentro del útero tiene esa edad y ese peso, contamos con la ultrasonografía como test, con sus naturales posibilidades de error.

\section{Aborto provocado: concepto}

Los conceptos son ideas abstraídas de la realidad. En esta solo existen numerosos casos, todos distintos entre sí, y nuestro entendimiento logra captar de toda esa particularidad de hechos individuales lo que unifica a algunos de ellos, constituyendo rasgos comunes o universales, como serían, por ejemplo, la movilidad y capacidad de desplazamiento de todos los mamíferos, y los rasgos necesarios, es decir, aquellos que los constituyen esencialmente, como la posesión de glándulas mamarias. Así el concepto y definición de "mamífero" tiene que incluir necesariamente la capacidad de lactar, pero no la de desplazamiento, ya que esta es común a muchos otros animales.

Si acercamos este ejemplo a las interrupciones del embarazo, es decir, a todas aquellas intervenciones que impiden que una gestación llegue a su término natural, e intentamos reconocer entre ellas las que corresponderían a un aborto provocado y las que no, tendríamos que fijarnos en aquellos ras- gos necesarios que distingan esencialmente unas de otras, y no en aquellos que, aunque fueran comunes, no lo hagan. Y lo que distingue esencialmente un aborto provocado de otras interrupciones, condición sine qua non, que por lo tanto debe siempre estar presente y que establece su concepto, es uno solo: la búsqueda intencionada de la muerte del embrión o feto. Pueden coexistir otros rasgos circunstanciales, como el método utilizado, pero que no permiten esa distinción, ya que se puede provocar un aborto mediante una cesárea y extraer un feto a las 38 semanas por esa vía, debido, por ejemplo, a una restricción severa del crecimiento intrauterino. Asimismo, es posible utilizar prostaglandinas para interrumpir una gestación cerca del término y también para provocar un aborto.

Una fuente de error es la confusión entre concepto y criterios; si definimos por los criterios, en realidad no definimos sino que apuntamos a cómo reconocer lo que queremos denotar ${ }^{6}$. Tampoco lo hacemos si fundamos el concepto en rasgos circunstanciales que, aunque pueden ser universales, no cumplen la necesidad que exige una definición.

Hay que tener presente que los rasgos circunstanciales en los abortos provocados no son tan ajenos a las acciones que los médicos habitualmente realizamos. Es perfectamente posible que una interrupción de embarazo con el objetivo que muera el feto utilice un método común y habitual en la práctica médica, como una inducción de actividad uterina con medicamentos, una rotura artificial de membranas o incluso una operación cesárea, prácticas todas utilizadas en clínicas abortivas legales en algunos países. La idea de la intención del que ejecuta la acción cobra entonces importancia.

Serán entonces los hechos externos asociados los únicos que podrán dar luces sobre las intenciones del médico en su intervención, estando ellas siempre ocultas en su interioridad. La manera de efectuar una interrupción y el trato otorgado al feto extraído en ciertas graves contingencias clíni-

${ }^{6}$ El hecho de que en algunos países exista el permiso legal de provocar la muerte fetal en edades gestacionales y pesos fetales mayores que la de los criterios actuales de viabilidad, refirma la tesis que la conducta abortiva está definida por la intención y no por los criterios de viabilidad. 
cas, podrán develar la radical diferencia entre una conducta abortiva y una intervención acorde a los fines y obligaciones de la medicina.

Existe otro aspecto de especial relevancia que será necesario precisar en orden de captar adecuadamente el concepto de "aborto provocado" y distinguirlo de otras interrupciones de embarazo o acciones que los médicos utilizamos para salvaguardar la vida y salud de nuestros pacientes. Podemos constatar en la práctica médica que algunas intervenciones nuestras culminan con la muerte del paciente. Estas muertes acaecidas durante procedimientos diagnósticos o terapéuticos pueden ser imputables a nuestro actuar, cuando no aplicamos adecuadamente las reglas de la profesión médica, por impericia, imprudencia o simplemente negligencia. Son muertes de las que, no estando en nuestra intención, somos igualmente responsables y merecemos tanto un juicio ético como uno penal.

Existen otras muertes de pacientes que tampoco están en nuestra intención y que ocurren como efecto secundario de intervenciones pensadas y planificadas. Esas muertes incluso pueden estar previstas como un riesgo inherente al procedimiento, pero la gravedad del paciente hace ver al clínico que el riesgo de no realizarlo es mayor o por lo menos igual que realizarlo. Son muertes que, a pesar de relacionarse con nuestra decisión e intervención, ocurren al intentar el médico evitar un daño mayor o equivalente (en estos casos lo único comparable sería el fallecimiento de alguien). Estas muertes son, entonces, indirectamente causadas por nuestra decisión y no son éticamente cuestionables, siempre que el balance entre el efecto que se busca sea proporcionado a la ocurrencia de ellas(7).

Para que constituya realmente una conducta abortiva, la muerte de un embrión o feto ocurrida bajo la acción de un médico no puede ser la muerte debida a un mal praxis, aunque ocurra antes de las 23 semanas o antes que el feto pese 500 gr. Esta muerte deberá ser juzgada no como un aborto provocado sino como negligencia, imprudencia o impericia con resultado de pérdida fetal. Tampoco será aborto provocado la muerte de un feto o embrión, incluso antes de cumplir los criterios actuales de viabilidad, que sea indirecta- mente causada por un médico al intentar este un efecto — aunque previsible- proporcionado.

La muerte del embrión o feto que cualifica una acción abortiva es la que se busca directamente, es aquella muerte que es el fin y propósito de la acción. Es aquella que si no ocurriera provoca frustración, y la acción por lo tanto se considera fracasada. Como vemos, esto no ocurre en la muerte debida a un mal praxis o como efecto colateral proporcionado. La prueba de ello es que lo único que quisiera el médico es que nunca hubiera ocurrido ${ }^{7}$.

De acuerdo con todas las consideraciones anteriores, la definición más adecuada de aborto provocado, la que apunta a los rasgos necesarios y no adventicios, sería la siguiente: "interrupción de un embarazo o acción que busca directamente la muerte del embrión o feto". Entendiendo que es independiente de la edad gestacional y peso fetal y, además, independiente del método que se utilice ${ }^{8}$.

\section{Aborto provocado y acción médica}

El establecimiento de la medicina por la escuela hipocrática, en el siglo $\mathrm{V}$ a.C., como un saber práctico fundado en la ciencia, diferente de otros saberes, como la filosofía, la religión y las ciencias naturales, tuvo como consecuencia, además, la constitución de un nuevo tipo de médico, un sujeto conocedor y poseedor de los conocimientos y destrezas necesarias para realizar la labor que se le encomendaba. Así, se obligaba a sí mismo al cumplimiento de su tarea de una manera acorde a la dignidad de ella. Nace así la profesión médica, con todo un bagaje de obligaciones intelectuales y de carácter moral, que rige sin grandes variaciones hasta nuestros días.

El fin de esta nueva profesión ha sido desde esos tiempos la prevención y la curación de la enfer-

\footnotetext{
7 Tanto califica la intención de que ocurra la muerte a una conducta abortiva, que incluso desde el punto de vista ético esta se establece, aunque la acción sea frustrada y no ocurra la muerte del feto. Todos los médicos obstetras conocemos estos casos y los denominamos "abortos frustros".

${ }^{8}$ Me parece que este concepto se debe circunscribir a los embriones implantados. Si bien es cierto, la occisión de un embrión pre-implantatorio es éticamente equivalente, la existencia de él no establece un embarazo.
} 
medad. Cuando hablamos de medicina, espontáneamente sabemos que nos referimos al cuidado de los enfermos. Incluso cuando se rastrean los orígenes de esta actividad, se buscan evidencias, en las antiguas tumbas, de señales en los restos corporales que muestren signos de intentos de curación. Así se describen piernas fracturadas que fueron alineadas, reducciones de luxaciones, o heridas tratadas satisfactoriamente. Siempre se ha entendido al médico como poseedor de conocimiento, teórico y práctico, que puede ser usado para tratar al enfermo(8).

Desde esta perspectiva debemos juzgar entonces las acciones que los médicos podemos y debemos realizar a nuestras pacientes embarazadas. Si nos corresponde, como obstetras, que una mujer y su hijo terminen su proceso de embarazo, parto y puerperio en las mejores condiciones de salud que nuestro estado del arte nos permite, si también nos corresponde como especialistas en medicina materno fetal el tratamiento de las patologías fetales, o de aquellas afecciones maternas en cuanto afectan el desarrollo de su hijo in utero, es que no podemos permitirnos, y tendríamos que rechazar como contrarios a los principios de nuestra especialidad y de nuestra profesión, cualquier intervención que busque intencionalmente atentar contra la integridad de ese ser humano en gestación?.

Podemos tratar a pacientes con gestaciones producto de una violación, otras con embarazos con fetos con alguna condición de pronóstico ominoso y también casos en que la mujer embarazada tiene una enfermedad o afección pero sin riesgo vital. Es decir, tenemos la posibilidad de decidir y realizar lo que se entiende por aborto "libre", "ético" o "terapéutico". Sin embargo, al reflexionar en lo que buscaríamos con esas interrupciones, nos damos inmediatamente cuenta que es lisa y llanamente que el feto sea eliminado. En el primer caso como intento de solución o medida paliativa para una paciente agredida brutalmente, en el segundo para aliviar a una madre de una situación angustiosa y en el tercero para solucionar

\footnotetext{
9 "El respeto de la vida humana desde su inicio y hasta su término constituye el fundamento básico del ejercicio profesional médico. Toda intervención médica realizada durante los nueve meses de gestación, deberá velar siempre por el mejor interés de la madre y del hijo.” Art. 8. Código de Ética. Colegio Médico de Chile A.G.
}

problemas que tienen verdaderos tratamientos médicos. Debemos recordar aquí que tenemos bajo nuestro cuidado a dos pacientes, y nuestra responsabilidad es que ambos terminen el proceso de embarazo de la mejor forma posible, y nos corresponde poner a su servicio todos los recursos razonables, toda nuestra ciencia y experiencia para velar por su salud.

Distinta evaluación debemos hacer en aquellas situaciones en que el médico tratante, después de hacer todos sus esfuerzos, llega a la convicción razonable que al continuar la gestación morirá la madre. En esos casos su obligación ética es interrumpir ese embarazo. De hecho, así lo consideramos universalmente los obstetras. Ahora bien, en vista de precisar la razón de esa legitimidad, tenemos que indicar que esa acción es lícita y obligatoria, no porque se está "privilegiando a la madre" o debido a que "es la única que se puede salvar". Es lícita y éticamente obligatoria para el tratante, debido a que es la única opción terapéutica también para el feto, ya que si se muere la madre inevitablemente también perecerá. En caso de ocurrir la muerte de él al nacer, sería una muerte lamentada por todos.

Es importante resaltar que, a pesar de la gravedad de estas situaciones, que demandan al tratante esmerarse en los cuidados para su paciente embarazada - intentando prolongar lo más posible la gestación para alcanzar así una mayor posibilidad para el recién nacido de vivir fuera del úterouna vez tomada la decisión, es decir, una vez que en él existe un íntimo convencimiento de que ya no puede hacer otra cosa, efectúa la intervención sin titubear. Todo eso es posible, ya que tiene la seguridad de que está cumpliendo su obligación ética y profesional. Quizá, y eso dependerá de la edad gestacional del feto y de las condiciones al nacer, pudiera ser que este no sobreviva, pero se le otorgarán los cuidados proporcionados, dependiendo estos de cada caso particular. Si sobrevive, todos se sentirán gratificados. Un médico prudente y bien preparado no puede omitir esa intervención; de hacerlo, implicaría dejar morir a sus dos pacientes sin intentar salvarlos. 


\section{Conclusión}

En el tema del aborto provocado y específicamente en el llamado aborto terapéutico, hay que ser muy cuidadoso. Es un tema en el que intervienen aspectos valóricos que surgen de posiciones antropológicas diversas. Además, es un problema en el cual todos tenemos derecho a opinión, ya que excede del ámbito privado de los ciudadanos y, por lo tanto, compromete el tipo de sociedad en la que queremos vivir. Es interesante y de especial relevancia incorporar a esa discusión la perspectiva de la profesión médica y específicamente la de los médicos, a quienes nos corresponde la atención de la mujer embarazada, por la responsabilidad que tenemos de efectuar las intervenciones sobre ellas y sobre el hijo que gestan.

La profesión médica, como toda profesión, tiene un compromiso hacia la sociedad. Este compromiso se vincula con aspectos de los que el médico tiene que hacerse cargo, y que tienen que corresponder no solo con las intervenciones que es capaz de realizar, sino que, con mayor relevancia, con las que se adecuan a los fines de la profesión médica. Los problemas que los pacientes presentan a la consideración de un médico han cambiado con los años, dependiendo de los avances del conocimiento científico y de las posibilidades tecnológicas. El médico no debe realizar intervenciones sin el consentimiento informado de su paciente, y no está obligado a realizarlas cuando considera que con ello vulnera los fines de la medicina. Habitualmente en la interacción médicopaciente esto no constituye un problema, ya que en la gran mayoría de los casos los objetivos del paciente y del médico coinciden. En escaso número de situaciones puede haber una dificultad, que se resuelve habitualmente exponiendo las razones de la petición por parte del paciente y las razones de la reticencia del clínico para efectuar algún procedimiento. Esto es lo que corresponde en una adecuada relación entre ambos.

La obstetricia y la medicina materno-fetal, en plena concordancia con los fines de la profesión médica, busca que una mujer embarazada y su hijo terminen el proceso de embarazo y parto en las mejores condiciones de salud posibles. Para ello se cuenta con numerosos recursos diagnósticos y terapéuticos. Uno de ellos es la interrupción del embarazo, cuando consideramos que continuar la gestación arriesga la salud de uno de ellos o de ambos. Siempre debemos ocupar esta herramienta terapéutica para esos fines y nunca buscando otros ajenos.

Sin duda este recurso se puede utilizar para resolver diversos otros problemas que alguna paciente puede presentar, algunos banales y otros secundarios a condiciones de extrema seriedad, pero en definitiva su utilización buscaría objetivos diametralmente opuestos al deber de un médico. Es el caso de interrumpir un embarazo o utilizar cualquier otro recurso con el fin de procurar la muerte de un embrión o feto.

Es fácil confundir lo que cada acción médica significa y muchas veces se oscurece la delimitación de si una acción realizada por un médico está en concordancia o no con lo que debe realizar. Para eso la precisión de los conceptos y acciones es fundamental.

Debemos entender como aborto provocado toda intervención que busca que un embrión o feto implantado sea eliminado. Esa intención es lo que especifica éticamente la acción.

Los criterios para establecer si una interrupción de un embarazo se adecua al concepto de aborto provocado no pueden ser entonces ni la edad gestacional ni el peso fetal. Si lo que establece el concepto de aborto provocado es la intención del médico, los únicos criterios son que este se responda las siguientes preguntas en cada caso particular: mi intervención ¿busca que este feto muera? ¿Estoy haciendo mis mejores esfuerzos para que sobreviva? Si la respuesta a la segunda pregunta es afirmativa, puede estar seguro que está efectuando una acción lícita y acorde a su deber como médico. Para un observador externo, y ya que las intenciones del médico están dentro de sus íntimos pensamientos, la manera como realiza la intervención puede dar luces e incluso servir como signo de que los criterios están presentes; el trato respetuoso al recién nacido y el otorgarle los cuidados proporcionados a su condición y dignidad reflejarán su verdadera intención, aunque su segundo paciente no logre sobrevivir. 
Las interrupciones del embarazo en la práctica obstétrica. Recurso terapéutico vs aborto provocado - Mauricio Besio

\section{Referencias}

1. Besio M. Aspectos bioéticos del aborto en Chile. Rev chil obstet ginecol 2013; 78(4): 256-258.

2. The Hastings Center. The Hasting Center Report, 1996: nov-dic.

3. Fernández C. Los filósofos medievales. Selección de textos. Vol. 1. Madrid: BAC; 1979: 545.

4. Besio M. La mujer gestante y su hijo. El médico frente al llamado aborto terapéutico. Ars Medica 2009; 18(18). Disponible en: http://escuela.med.puc.cl/publ/arsmedica/ArsMedica18/Mujer.html.

5. Bernat J, Culver C. On de Definition and Criterion of Death. Annals of Internal Medicine 1981; 94: 389-394.

6. Taboada P. El derecho a morir con dignidad. Acta Bioethica 2000; 6(1): 89-101.

7. Miranda A. ¿Qué es el aborto indirecto? La prohibición del aborto y el principio del doble efecto. Acta Bioethica 2014; 20(2): 159-168.

8. The Cambridge Illustrated History of Medicine. Cambridge University Press; 1996.

Recibido: 2 de diciembre de 2014

Aceptado: 20 de enero de 2015 